\title{
The morphology of cometary X-ray emission
}

\author{
R. Wegmann ${ }^{1}$, K. Dennerl ${ }^{2}$, and C. M. Lisse ${ }^{3}$ \\ 1 Max-Planck-Institut für Astrophysik, 85748 Garching, Germany \\ e-mail: ruw@mpa-garching.mpg.de \\ 2 Max-Planck-Institut für extraterrestrische Physik, 85748 Garching, Germany \\ e-mail: kod@mpe.mpg.de \\ 3 University of Maryland, Department of Astronomy, College Park, MD 20742, USA \\ e-mail: lisse@astro.umd.edu
}

Received 2 April 2004 / Accepted 10 August 2004

\begin{abstract}
X-ray emission from comets is generated by charge exchange of heavy solar wind ions in a high ionization state with the neutral particles of the cometary coma. We use model calculations and theoretical considerations to study the morphology of the emission. The emission is located in a bowl-shaped region on the subsolar side of the nucleus. We derive several length scales. By comparison with hydrodynamic scales we find that the maximum emission comes from inside the bow shock. The size of the emission region depends on the size of the cometary coma, i.e., mainly on the gas production, while the intensity depends mainly on the energy content of the heavy ions in the solar wind flow. This dependence allows a determination of the gas production of the comet and the heavy ion energy flux from a statistical study of the observed spatial distribution of X-ray photons. Here we apply this procedure to X-ray observations from comets C/1996 B2 (Hyakutake), C/1990 K1 (Levy), 2P/Encke and C/2000 WM1 (LINEAR). The gas production rates determined by our method agree very well with those obtained by other methods. The variability of the emission documented in several recorded outbursts correlates with the solar wind proton density.
\end{abstract}

Key words. comets: general - Sun: solar wind - X-rays: general

\section{Introduction}

The X-ray emission from comet C/1996 B2 Hyakutake detected by the Röntgen satellite ROSAT (Lisse et al. 1996) has been successfully explained as an interaction by charge exchange of heavy solar wind ions in a high ionization state with the neutral particles in the cometary coma (Cravens 1997). Model calculations demonstrated that this mechanism explains very well the total intensity, the morphology of the emitting region, and the spectrum of the radiation (Häberli et al. 1997; Wegmann et al. 1998).

There have now been several more X-ray observations of comets. The review of Lisse et al. (2004) lists observations of 18 comets. With ROSAT the comets C/1996 B2 Hyakutake (Lisse et al. 1996), 2P/Encke (Lisse et al. 1999), and C/1996 Q1 Tabur (Dennerl et al. 1997) were observed, with Chandra the comets C/1999 S4 (LINEAR) (Lisse et al. 2001) and C/1999 T1 McNaught-Hartley (Krasnopolsky et al. 2002), and with XMM-Newton the comet C/2000 WM1 (LINEAR) (Dennerl et al. 2003). Cometary traces in the ROSAT AllSky Survey came to the observers' attention only years later (Dennerl et al. 1997). Comet C/1995 O1 Hale-Bopp was observed with Bepposax (Owens et al. 1998). Soft X-rays where detected with EUVE from several comets (Mumma et al. 1997; Krasnopolsky et al. 2000). In addition there are a few still unpublished observations. It is now obvious that most (if not all) comets emit X-rays. This opens a new observational window. The question arises of how to use the X-ray data to retrieve information about the comet, about the solar wind, and about details of the charge exchange mechanism. This paper is a first step in this direction.

The plan of the paper is as follows. In Sect. 2 we derive, using a simple physical model, a scale length for the X-ray emission. In Sect. 3 we describe how the hydrodynamic flow, in particular the cometary bow shock, modifies the cometary emission region. A comparison of scales shows that the maximum of the emission always comes from well inside the bow shock. We show how the emitting region scales with cometary parameters such as shock distance and gas production rate. In Sect. 4 we demonstrate how the morphology depends on the phase angle. In Sect. 5 we show how the observed morphology of the cometary X-ray emission depends also on the spectral range of the observing instrument. In Sect. 6 we describe a maximum likelihood method which allows us to derive the gas production rate from X-ray observations of a comet at known phase angle and instrument sensitivity. Since the gas 
production is determined from the morphology alone, the intensity gives independent information about the heavy ion flux in the solar wind. We apply the method in Sect. 7 to several comets and compare our estimated gas production rates with the results of other observers obtained by different techniques at longer wavelengths. In Sect. 8 we correlate our estimates for the solar wind heavy ion energy flux with the solar wind proton density. This gives some evidence for the causes of the observed outbursts. In Sect. 9, the X-ray background estimated by our analysis is compared with the observed background. In Sect. 10 we show that the total X-ray luminosity of a comet scales with the square of the gas production rate. In the final section, Sect. 11, we discuss our results and draw some conclusions from our work.

Our modeling is based on a continuum description of the neutral coma as well as the plasma flow. As the examples discussed in our paper show, this approach works very well for comets with outgassing rates larger than about $10^{28}$ particles per second. Comets with low outgassing rates may become "collisionally thin" for the solar wind heavy ions due to the finite number of cometary particles which decreases with the outgassing rate and finally invalidates a continuum description of the interaction.

\section{Simple physical model}

When the solar wind penetrates the neutral cometary coma, the highly ionized heavy ions acquire more and more electrons by successive charge exchange events. The new electrons are first captured in shells with principal quantum number of around 4 to 5 . When they de-excite to lower energies, line radiation is emitted. This radiation is in the energy range of several hundreds of eV for highly ionized atoms of oxygen, carbon, nitrogen, neon, magnesium, silicon, sulfur and iron (Wegmann et al. 1998; Kharchenko \& Dalgarno 2000, 2001). Since the neutral gas density varies as $R^{-2}$ with the distance $R$ from the nucleus, the ions arriving along the Sun-Comet line meet sufficiently many neutral particles to become completely neutralized. The region close to the nucleus is collisionally thick for the ions. On the other hand at a certain distance the heavy ions have a good chance of passing through the tenuous neutral coma unimpeded. This region is collisionally thin for the ions. Thus, charge exchange happens most efficiently in a bowl-shaped region which envelopes the nucleus on the subsolar side. There is no emission in the central part of the tail.

A rough qualitative picture of the X-ray emission can be obtained by a simple model.

From the cometary nucleus gas sublimates and streams off with a velocity $w$. The gas forms a spherically symmetric neutral gas cloud with density

$N_{n}=\frac{G}{4 \pi w R^{2}}$

proportional to the total gas production $G$ and to the inverse square of the distance $R$. The solar wind arrives at the comet as a parallel flow with velocity $u_{\odot}$. It carries heavy ions with a density $N_{i, \odot}$. The heavy ions and the cometary neutrals interact by charge exchange with a cross-section $Q$. The heavy ions

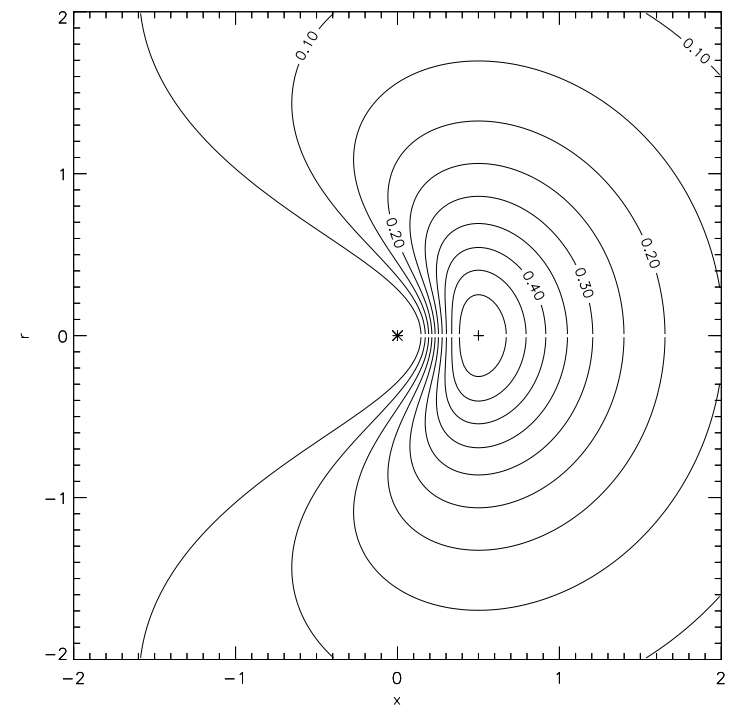

Fig. 1. Simple model: the volume intensity $I$ in a meridional plane for $H=1$ and $l_{x}=1$. The nucleus is marked by a star and the point of maximum emission by a plus.

are consumed by this process. For the simple model we assume that the solar wind remains a parallel flow and retains its velocity $u_{\odot}$. Then the heavy ion density $N_{i}$ satisfies the differential equation

$\frac{\mathrm{d} N_{i}}{\mathrm{~d} t}=-Q N_{i} N_{n} u_{\odot}$

where $N_{i} u_{\odot}$ is the solar wind heavy ion number flux. Let $x$ be the coordinate along the Sun-Comet line, positive towards the Sun. Then $u_{\odot}=-\mathrm{d} x / \mathrm{d} t$ and, using expression (1), Eq. (2) can be transformed to

$\frac{1}{N_{i}} \frac{\mathrm{d} N_{i}}{\mathrm{~d} x}=\frac{l_{x}}{R^{2}}$

This equation contains only a single parameter namely the length scale $l_{x}$ given by

$l_{x}=\frac{G Q}{4 \pi w}$.

In cometocentric cylindrical coordinates, $x=R \cos \theta, r=$ $R \sin \theta$, the differential Eq. (3) has the solution

$N_{i}=N_{i, \odot} \exp \left(-\frac{\theta}{\sin \theta} \frac{l_{x}}{R}\right)$

When each charge exchange process emits a photon of average energy $E$ the volume intensity of the X-ray emission is

$I=Q N_{i} N_{n} u_{\odot} E=\frac{u_{\odot} N_{i, \odot} E}{l_{x}} \exp \left(-\frac{\theta}{\sin \theta} \frac{l_{x}}{R}\right)\left(\frac{l_{x}}{R}\right)^{2}$.

The volume intensity is proportional to the solar wind heavy ion energy flux density

$H=u_{\odot} N_{i, \odot} E$

and inversely proportional to the length scale $l_{x}$. Figure 1 shows the volume intensity from this simple model in a meridional 
plane calculated for unit solar wind heavy ion energy flux density $H=1$ and unit length scale $l_{x}=1$.

The simple functional dependence of Eq. (6) describes an emission region that envelops the comet like a bowl on the subsolar side. There is no emission from the negative $x$-axis in the shadow of the comet as the argument of the exponential goes to $-\infty$ for $\theta \approx \pi$. Maximum intensity $I_{\max }=4 H /\left(e^{2} l_{x}\right)$ occurs on the Sun-Comet line $(\theta=0)$ at a distance $x_{\max }=l_{x} / 2$.

Equation (6) can be interpreted in two different ways which must not be confused: at a fixed point in space with polar coordinates $(x, r)$ the volume intensity is proportional to $l_{x}$, i.e., a comet with low activity has at this point less X-ray intensity than a comet with high activity. On the other hand at the properly scaled points with coordinates $\left(x / l_{x}, r / l_{x}\right)$ the $\mathrm{X}$-ray volume intensity is proportional to $1 / l_{x}$, i.e., the comet with small outgassing rate is brighter in X-rays than the comet with high outgassing rate. This becomes manifest in the maximum volume intensity $I_{\max }$ which is proportional to $1 / l_{x}$.

$\mathrm{X}$-ray satellites observe surface intensities, i.e., the volume intensities integrated along the lines of sight. It follows from the representation (6) of the volume intensity that the surface intensity observed under a phase angle $\alpha$ (=angle observer Comet - Sun) at a position $(x, y)$ in cometocentric coordinates has a representation of the form

$J_{\alpha}(x, y)=u_{\odot} N_{i, \odot} E F_{\alpha}\left(\frac{x}{l_{x}}, \frac{y}{l_{x}}\right)$

with a function $F_{\alpha}(\xi, \eta)$ independent of the comet and the solar wind. The spatial morphology is only determined by the length scale $l_{x}$ and the phase angle $\alpha$. The intensity is proportional to the solar wind heavy ion energy flux density $H$.

It follows from Eq. (6) that for large distances $R$ the intensity behaves like

$I \approx \frac{u_{\odot} N_{i, \odot} E l_{x}}{R^{2}}$

everywhere except in the tail region where $\theta$ is close to $\pi$. Integration along the line of sight shows that the surface intensity

$J(x, y) \approx \frac{A}{R}$

is inversely proportional to the distance $R=\sqrt{x^{2}+y^{2}}$ from the nucleus with the constant

$A=\pi u_{\odot} N_{i, \odot} E l_{x}=\frac{H G Q}{4 w}$.

This result is independent of the phase angle $\alpha$. It is remarkable that the comet is surrounded by an X-ray halo with intensity falling off with the inverse of the distance - the same result obtained for scattering of sunlight in a spherically symmetric coma. The intensity is proportional to the product $H G$ of the solar wind heavy ion energy flux density $H$ and the total gas production $G$. From the behaviour of the $\mathrm{X}$-rays at large distances we cannot get information on $H$ and $G$ separately.

For comet Hyakutake in late March $1996(G=$ $\left.10^{29} \mathrm{~s}^{-1}, Q=5 \times 10^{-15} \mathrm{~cm}^{2}, w=10^{5} \mathrm{~cm} \mathrm{~s}^{-1}\right)$ the length scale is $l_{x}=4 \times 10^{8} \mathrm{~cm}$. This is much smaller than the distance, $\approx 2 \times 10^{9} \mathrm{~cm}$, of the observed peak brightness. This shows that the simple model is inadequate. The flow of the cometary plasma, in particular the bow shock, must be included into the model.

\section{Hydrodynamic models}

A more sophisticated treatment of the cometary X-ray problem is possible.

The interaction of the solar wind with a comet has been investigated by many hydro- and magnetohydrodynamic model calculations. The theoretical results have been confirmed by in situ measurements at several comets. This interaction is now well understood. Molecules and atoms sublimate from the cometary nucleus and stream off radially with a constant velocity $w$. They are ionized by various processes and added to the solar wind plasma.

The interaction can be described by hydrodynamic or magnetohydrodynamic equations with source terms. The source terms describe the effect of the pick-up of newly generated cometary ions. The solar wind as a parallel flow can only digest a certain amount of cometary ions. When the critical mass is exceeded a bow shock occurs which transforms the parallel supersonic solar wind flow into a divergent subsonic flow. A lower bound for the shock distance $R_{\mathrm{S}}$ is given by

$R_{\mathrm{S}}>\left(\gamma^{2}-1\right) R_{I}$

where $\gamma$ is the adiabatic index (usually $\gamma=5 / 3$ ) and $R_{I}$ is the interaction length scale

$R_{I}=\frac{\sigma_{\mathrm{av}} m_{C} G}{4 \pi w \rho_{\odot} u_{\odot}}$

with the solar wind mass density $\rho_{\odot}$ and velocity $u_{\odot}$, the average mass of a cometary ion $m_{C}$ and the average ionization rate $\sigma_{\mathrm{av}}$ (Schmidt \& Wegmann 1982). The ratio of the length scales (4) for X-ray emission and (13) for hydrodynamic interaction

$\frac{l_{x}}{R_{I}}=\frac{Q \rho_{\odot} u_{\odot}}{m_{C} \sigma_{\mathrm{av}}}$

is independent of the cometary outgassing parameters $G$ and $w$. For typical solar wind conditions with $\rho_{\odot}=10 \mathrm{~m}_{\mathrm{p}} \mathrm{cm}^{-3}, u_{\odot}=$ $4 \times 10^{7} \mathrm{~cm} / \mathrm{s}$ and typical values $m_{C}=20 m_{\mathrm{p}}, \sigma_{\mathrm{av}}=10^{-6} \mathrm{~s}^{-1}$, $Q=5 \times 10^{-15} \mathrm{~cm}^{2}$, this ratio has the value 0.1 . This means that $l_{x}$ is an order of magnitude smaller than the distance of the bow shock.

If the maximum X-ray emission would come from outside the bow shock, the point of maximum brightness could be determined by the simple model of Sect. 2. The maximum would be at a distance $l_{x} / 2$. For any reasonable solar wind conditions, however, this distance is much smaller than the shock distance. Therefore, the point of maximum X-ray brightness must lie well inside the bow shock. We must consider the plasma flow in more detail in order to describe the X-ray emission adequately.

We have calculated several hydrodynamic models with a simplified chemistry as described by Wegmann et al. (1999). The heavy solar wind ions are treated as test particles which are 


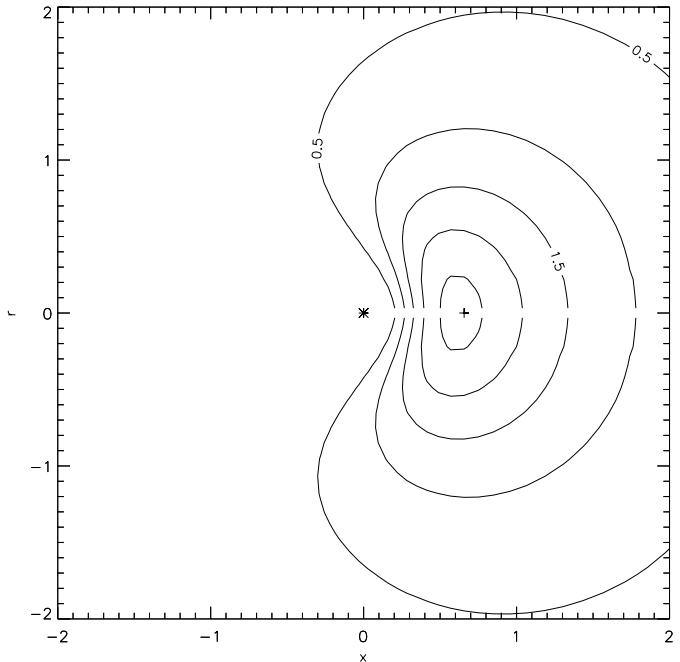

Fig. 2. Hydro model: the volume intensity $\left[10^{-15} \mathrm{ergs} \mathrm{s}^{-1} \mathrm{~cm}^{-3}\right]$ of the X-ray emission in a meridional plane for the model with $G=3 \times$ $10^{29} \mathrm{~s}^{-1}$ and fast solar wind. The length is in units of $10^{5} \mathrm{~km}$. The nucleus is marked by a star and the point of maximum emission by a plus.

passively convected in the flow of the hydro models. Models were produced for the three production rates $G=1,3$, and $10 \times$ $10^{29} \mathrm{~s}^{-1}$, and for slow and fast solar wind conditions with heavy ion content as described by Schwadron \& Cravens (2000). The flow parameters are $\rho_{\odot}=7 \mathrm{~m}_{\mathrm{p}} \mathrm{cm}^{-3}, u_{\odot}=400 \mathrm{~km} \mathrm{~s}^{-1}$ for slow and $\rho_{\odot}=4 m_{\mathrm{p}} \mathrm{cm}^{-3}, u_{\odot}=750 \mathrm{~km} \mathrm{~s}^{-1}$ for fast solar wind.

The heavy solar wind ions are convected with the velocity field $\boldsymbol{u}$ of the cometary plasma. They interact by charge exchange with the neutral particles with a cross-section $Q_{k}$ depending on the ion species $k$. This process releases a photon of energy $E_{k}$ (see Wegmann et al. 1999, and Schwadron \& Cravens 2000 for a list of values of $Q_{k}$ and $E_{k}$ ). The effective velocity $v_{\text {eff }}$ for the interaction is a combination of the convective and thermal velocities of the cometary plasma particles. The density $N_{k}$ of the $k$-th heavy ion species satisfies the stationary continuity equation

$$
\operatorname{div}\left(N_{k} \boldsymbol{u}\right)=-Q_{k} N_{k} N_{n} v_{\text {eff }} .
$$

The volume intensity of the X-ray emission is

$I=\sum_{k} Q_{k} N_{k} N_{n} v_{\mathrm{eff}} E_{k}$

The sum is over all ion species $k$ which emit by charge exchange a photon with energy $E_{k}$ in the X-ray range. Figure 2 shows the intensity of the X-ray emission in a meridional plane for one of the models.

We derive now a lengthscale for the $\mathrm{X}$-ray emission in the hydro models. We assume for the estimates that $\gamma=5 / 3$; we also assume a strong shock with solar wind Mach number $M a_{\odot}=\infty$. In a shock of this kind the velocity is reduced by a factor 4 . Behind the bow shock the velocity decreases linearly with distance $u=0.25 u_{\odot} R / R_{\mathrm{S}}$. The effective velocity for collisions is a combination of flow and thermal velocity. It remains nearly constant at $v_{\text {eff }} \approx 0.77 u_{\odot}$ for a long time until it

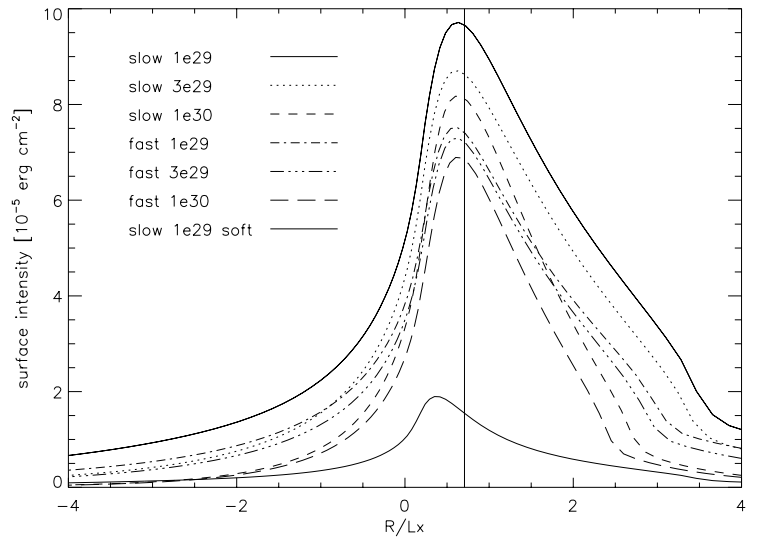

Fig. 3. The surface intensity along the Sun-Comet line seen under aspect angle $90^{\circ}$ for different models. The models are identified by the state of the solar wind and the gas production (e.g. "slow 1e29" means slow solar wind and $G=10^{29} \mathrm{~s}^{-1}$ ). The distance is scaled with $L_{x}$ as defined in Eq. (18). The vertical line marks the distance $X_{\max }$ of maximal volume intensity defined by Eq. (20). The lowest line (from a $E<200 \mathrm{eV}$ model) will be explained in Sect. 5 .

decreases near the nucleus due to cooling by pick-up ions. With these values of the velocities, Eq. (3) is modified to

$\frac{1}{N_{i}} \frac{\mathrm{d} N_{i}}{\mathrm{~d} x}=Q \frac{v_{\mathrm{eff}}}{u} N_{n}=\frac{L_{x}^{2}}{R^{3}}$

with a new length scale

$L_{x}=1.75 \sqrt{l_{x} R_{\mathrm{S}}}$.

The density is compressed in the shock by a factor of 4 . Hence Eq. (17) must be integrated with the initial value $N_{i}=4 N_{i, \odot}$ at the shock. This gives the solution

$N_{i}=4 N_{i, \odot} \exp \left(-\frac{L_{x}^{2}}{x^{2}}\right)$

for the heavy ion density $N_{i}$ along the line connecting the subsolar points of the bow shock and the contact discontinuity. The maximum luminosity occurs along the Sun-Comet line at a distance of

$X_{\max }=L_{x} / \sqrt{2}$

from the comet. Using the estimates (12) and (14) we find that this distance is about $5 \cdot l_{x}$. This is an order of magnitude larger than the distance $l_{x} / 2$ obtained for the simple model in Sect. 2 . Hence it is much closer to the observed distance of maximum brightness for comet Hyakutake (Lisse et al. 1996). The maximum volume intensity is

$I_{\max }=0.74 \frac{H}{R_{\mathrm{S}}}$.

For the example shown in Fig. 2 the values are: $R_{\mathrm{S}}=2.9 \times$ $10^{5} \mathrm{~km}, L_{x}=1.03 \times 10^{5} \mathrm{~km}, X_{\max }=7.3 \times 10^{4} \mathrm{~km}, I_{\max }=$ $2.78 \times 10^{-15} \mathrm{ergs} \mathrm{s}^{-1} \mathrm{~cm}^{-3}$. In the model the maximum volume brightness of $2.65 \times 10^{-15} \mathrm{ergs} \mathrm{s}^{-1} \mathrm{~cm}^{-3}$ occurs at a distance of $6.6 \times 10^{4} \mathrm{~km}$.

Figure 3 shows the surface intensity along the Sun-Comet line for several models as a function of distance. The distance 


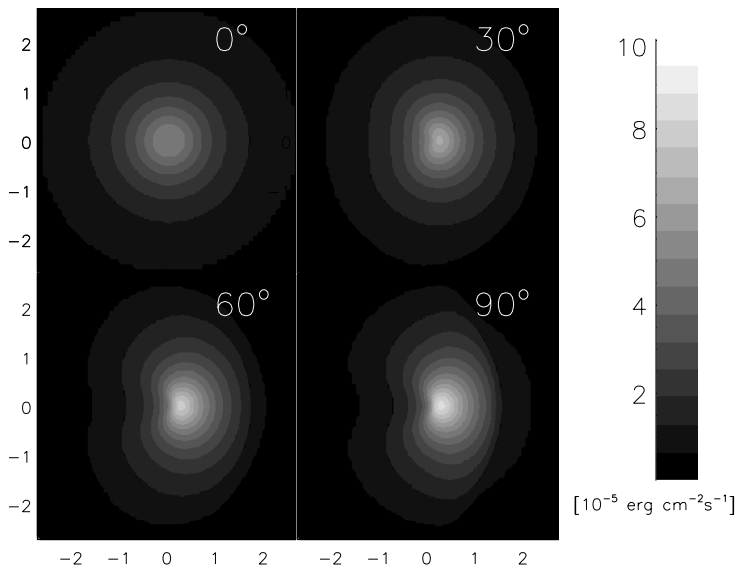

Fig. 4. The X-ray emission of a comet with gas production $G=$ $10^{29} \mathrm{~s}^{-1}$ and slow solar wind observed with phase angles: $0,30,60$, $90^{\circ}$. The length scale is $10^{5} \mathrm{~km}$. The Sun is to the right in each image.

is measured by the scale $L_{x}$. All curves look similar and have their maximum close to the estimate (20) for the distance $X_{\max }$ of maximal volume intensity.

The average energy content $E$ of a heavy ion in the solar wind is $216 \mathrm{eV}$ for fast and $315 \mathrm{eV}$ for slow solar wind (Schwadron \& Cravens 2000). This difference is due to the fact that the fast wind originates in coronal holes, which are cooler than the average solar corona. The solar wind heavy ion energy flux used in our models is $H=1.55 \times 10^{-4} \mathrm{erg} \mathrm{cm}^{-2} \mathrm{~s}^{-1}$ for the slow, and $H=1.10 \times 10^{-4} \mathrm{erg} \mathrm{cm}^{-2} \mathrm{~s}^{-1}$ for the fast solar wind. This difference explains why the intensities are lower for models with fast solar wind than those with slow solar wind. The intensities also decrease with increasing coma size of the comet. This can be explained by the exhaustion of the neutrals by ionization: not all neutral particles reach the region where most of the X-rays are generated, but some are ionized earlier and swept away by the solar wind.

In any case for a given comet and given solar wind type, the intensity is proportional to the solar wind heavy ion energy flux $H=u_{\odot} N_{i, \odot} E$. We have inserted in Fig. 3 the values from a "soft" model, which will be explained in Sect. 5.

The length scale $L_{x}$ depends on several cometary and solar wind parameters. Since $l_{x} \propto G$ and $R_{\mathrm{S}}$ is roughly proportional to $R_{I} \propto G$, it follows from Eq. (18) that $L_{x} \propto G$. Therefore, for practical purposes it is sufficient to say that $L_{x}$ is proportional to the shock distance $R_{\mathrm{S}}$ or that it is proportional to the production rate $G$. We will use this result later in Sects. 7 and 10 .

\section{Morphological dependence on the phase angle}

We have seen in Sects. 2 and 3 that the X-rays come from a bowl-shaped region which envelops the nucleus on the subsolar side. The comet is optically thin for the emitted X-rays. In projection, the bowl-shaped emission region looks different when observed from different directions. The observed surface intensities are volume intensities integrated over the lines of sight.

We present in Fig. 4 images of the X-ray emission of a model comet with gas production of $G=10^{29} \mathrm{~s}^{-1}$ and slow

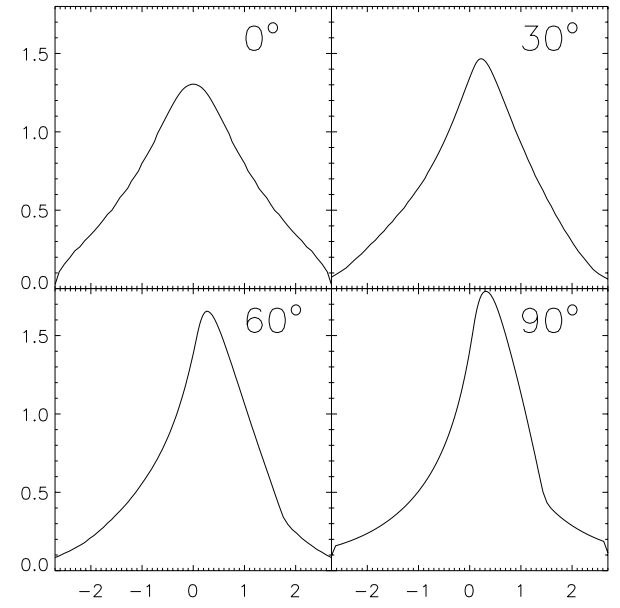

Fig. 5. The X-ray emission of Fig. 4 integrated over lines perpendicular to the Sun-Comet-line. The length scale is $10^{5} \mathrm{~km}$. The unit is $10^{6} \mathrm{erg} \mathrm{cm}^{-1} \mathrm{~s}^{-1}$.

solar wind for different phase angles $\alpha$ (=angle observer Comet - Sun).

For $\alpha=0^{\circ}$ the convex side of the bowl is seen from above. The image is rotationally symmetric. In the collisionally thick center the ions release all their energy, while in the collisionally thin outer part a decreasingly smaller fraction of the available recombination energy is transformed into $\mathrm{X}$-ray radiation.

At a phase angle of $\alpha=30^{\circ}$ the image becomes noticeably asymmetric. The outer contour lines are elongated to ovals and are shifted sunward. The point of maximal brightness is also shifted sunward to a distance of $23000 \mathrm{~km}$. For $\alpha=60^{\circ}$ the image resembles a crescent. The point of maximal brightness is about $26000 \mathrm{~km}$ in front of the nucleus. The view from the side $\left(\alpha=90^{\circ}\right)$ reveals the most details. In particular, at a distance of $1.4 \times 10^{5} \mathrm{~km}$ the bow shock can be recognized as a rather narrow transition from low to high intensity. This is an imprint of the bow shock, where the ions are compressed by a factor of 4 and heated. Although the flow velocity is reduced by a factor of 4 , the effective velocity $v_{\text {eff }}$ is only reduced to $77 \%$ of the pre-shock value. Therefore, the probability of charge exchange is enhanced in the shock by a factor of about 3 .

Figure 4 shows also that the maximum observed intensity depends on the phase angle. The values for the four angles $\alpha=$ $0,30,60,90^{\circ}$ are 5.6, 7.2, 8.7, and $9.4 \times 10^{-5} \mathrm{erg} \mathrm{cm}^{-2} \mathrm{~s}^{-1}$.

By integration over lines perpendicular to the Sun-Cometline we get intensities per unit length. Figure 5 shows how these integrated intensities depend on the phase angle. For $\alpha=0^{\circ}$ the curve is broad and symmetric. With increasing phase angle it becomes more and more asymmetric, with the peak shifted towards the sun. When viewed from the side $\left(\alpha=90^{\circ}\right)$, the integrated intensity drops very sharply at a distance of around $1.4 \times 10^{5} \mathrm{~km}$. This is an imprint of the bow shock.

\section{Morphological dependence on the spectral range}

The heavy solar wind ions recombine with electrons captured from neutral species in the cometary coma. First they catch one 


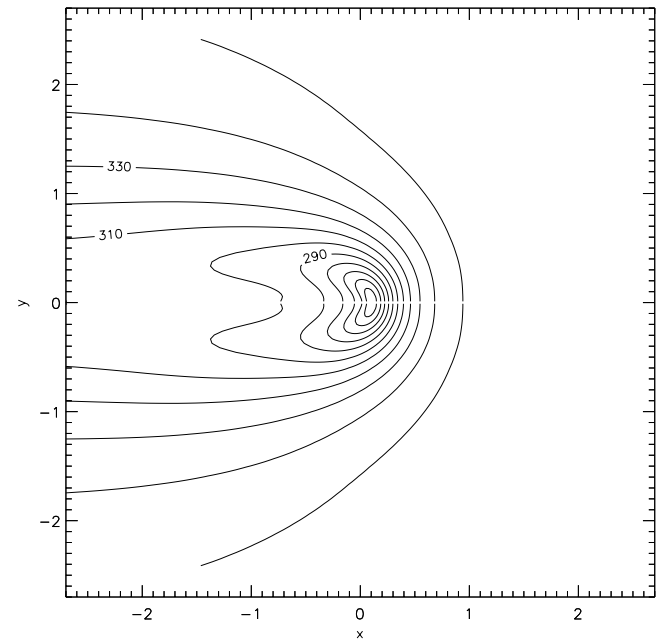

Fig. 6. The average energy (measured in $\mathrm{eV}$ ) of the photons of the $\mathrm{X}$-ray emission of a comet with gas production $10^{29} \mathrm{~s}^{-1}$ observed with phase angle $90^{\circ}$. The length scale is $10^{5} \mathrm{~km}$.

electron. While they remain in a sufficiently dense part of the coma, they can capture another electron, and so on. The ions passing through the central part of the coma fall through a cascade of states. In each charge exchange process they emit a photon, which in the first few steps is still in the X-ray or extreme UV range. For the lower ionization states, however, the wavelength increases. The completely ionized oxygen emits in the first steps of the cascade $\mathrm{O}^{8+} \rightarrow \mathrm{O}^{7+} \rightarrow \mathrm{O}^{6+} \rightarrow \mathrm{O}^{5+} \rightarrow$ $\mathrm{O}^{4+} \rightarrow \mathrm{O}^{3+}$ photons of energy 837, 709, 103, 63 and $43 \mathrm{eV}$, respectively. (We assumed here that the captured electron first occupies the state with principal quantum number $n$ calculated with the formula used by Wegmann et al. 1998.) As a consequence, in the inner coma there are not only the $\mathrm{O}^{6+}$ ions injected by the solar wind but also the $\mathrm{O}^{6+}$ ions generated during the recombination cascade from $\mathrm{O}^{8+}$ and $\mathrm{O}^{7+}$. These $\mathrm{O}^{6+}$ ions emit in a further charge exchange process photons of energy $103 \mathrm{eV}$. The further steps $\mathrm{O}^{3+} \rightarrow \mathrm{O}^{2+} \rightarrow \mathrm{O}^{1+} \rightarrow \mathrm{O}$ of the cascade are not associated with $\mathrm{X}$-ray radiation since the captured electron populates the state with minimal quantum number $n=2$.

In the outer part of the coma the high ionization states recombine and emit (relatively) hard X-ray photons with energies of $400 \mathrm{eV}$ and more. In the inner part there is an enrichment of lower ionization states, which emit soft X-rays and extreme UV radiation with photon energies of $200 \mathrm{eV}$ and less.

In view of this ion distribution the spectrum of the cometary $\mathrm{X}$-ray emission varies across the comet. The spectrum is hardest in the outer part where the pure solar wind first impinges on the coma. It becomes increasingly softer in the inner coma. As a measure of "hardness" we use the average energy of the emitted photons. We show in Fig. 6 the average photon energy emitted by a model comet with gas production $G=10^{29} \mathrm{~s}^{-1}$ and slow solar wind. The emission is integrated along lines of sight, so that the image represents the situation as it would be observable from a satellite in Earth orbit. The average energy ranges from $244 \mathrm{eV}$ in the subsolar crescent to $360 \mathrm{eV}$ in the outer coma close to the undisturbed solar wind.
There is another observable consequence of the spatial variation of the spectrum. When the comet is observed with an instrument which is sensitive only to relatively soft X-ray photons then the hard photons from the distant part are not registered. Therefore, in an instrument measuring only soft X-rays, the comet seems to be less bright, since the hard part of the emission is neglected. The emission, however, apparently also has a smaller spatial extent than in an observation which registers all X-ray photons. This can be demonstrated by model calculations. To this aim we have added to Fig. 3 the surface intensity along the Sun-Comet line from a model with $G=10^{29} \mathrm{~s}^{-1}$ and slow solar wind, where only the photons with energy less than $200 \mathrm{eV}$ are taken into account. The peak intensity is reduced by a factor of 5 compared with the full model. In addition, the point of maximal brightness is shifted from a distance of $26000 \mathrm{~km}$ to a distance of $14500 \mathrm{~km}$, i.e., this distance is reduced by almost a factor of 2 .

Comet Hyakutake was observed with the ROSAT widefield camera (WFC) which was sensitive in an energy range between 90 and $206 \mathrm{eV}$ (Lisse et al. 1996). There are simultaneous observations with the high-resolution imager (HRI). It is interesting to compare the observations of the two instruments.

In Fig. 7 we show the simultaneous observations of comet Hyakutake on March 27.57 1996. The images look quite similar. The maximum emission is on a crescent on the subsolar side of the nucleus. The crescent of the WFC observations, however, seems to be closer to the nucleus than the HRI crescent. This becomes even more obvious in the intensities averaged over lines perpendicular to the Sun-Comet line. The maximum for WFC is at a distance of $24500 \mathrm{~km}$, while the HRI data have their maximum near $37000 \mathrm{~km}$ distance. These numbers are derived from the smooth curves in the upper left panels of Figs. 9 and 10.

Another quantity measuring the distance of the crescent is the average $\bar{x}$ of the coordinate $x$ weighted with the intensity over the field shown in Fig. 7. We get $\bar{x}_{\mathrm{WFC}}=16600 \mathrm{~km}$ for WFC, and $\bar{x}_{\mathrm{HRI}}=19900 \mathrm{~km}$ for HRI. The relation $\bar{x}_{\mathrm{WFC}}<$ $\bar{x}_{\text {HRI }}$ is found for all ROSAT observations of comet Hyakutake in March 1996. These pairs of WFC and HRI observations thus confirm nicely the spectral variation across the image predicted by the model calculations.

\section{Parameter estimation}

The detector in an X-ray satellite registers single photons, their time of arrival, and the place in the sky where they come from. Figure 8 shows in a cometocentric coordinate system $\boldsymbol{x}=(x, y)$ the positions of the X-ray photons from Comet Hyakutake by the ROSAT Wide field camera on March 27.57 1996. The field of view is a disc with radius of about $730000 \mathrm{~km}$. The smaller square contains $100 \times 100$ pixels and has side length $5.5 \times 10^{5} \mathrm{~km}$.

The photons are emitted randomly. The probability $p(\boldsymbol{x})$ that a photon will be registered at a position $\boldsymbol{x}$ is proportional to the surface intensity $J_{\alpha}(\boldsymbol{x})$ of the comet interacting with the solar wind seen under a phase angle $\alpha$ and to the exposure time $T(\boldsymbol{x})$. This simple picture is disturbed by the presence of other X-ray sources in the field of view, by 


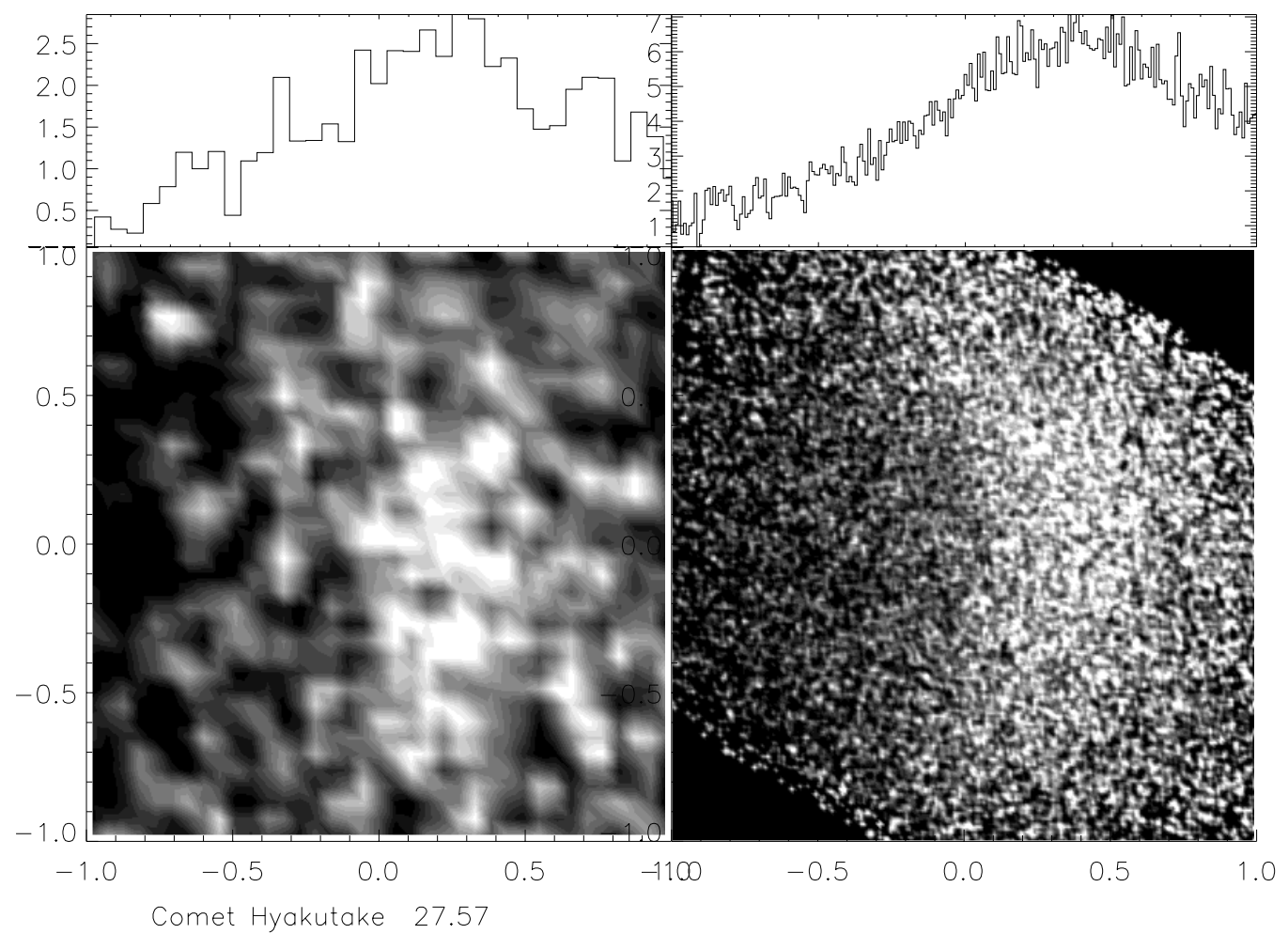

Fig. 7. The intensity of the X-ray emission observed on March $27.57,1996$ from comet Hyakutake (smoothed) and the intensity $\left[10^{-5} \mathrm{erg} / \mathrm{cm}^{2} / \mathrm{s}\right]$ averaged over lines perpendicular to the Sun-Comet line (top panel). On the left the WFC data, on the right the HRI data. The length scale is $10^{5} \mathrm{~km}$.

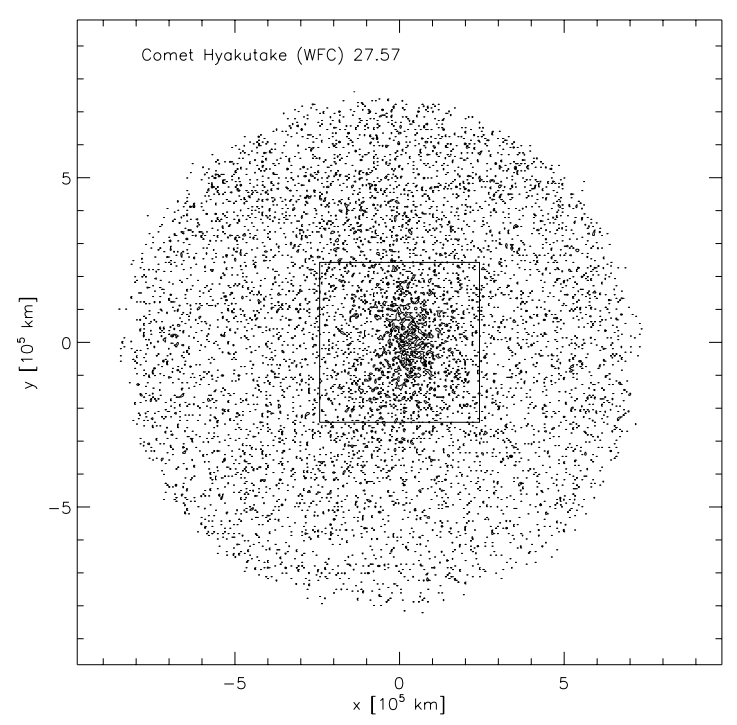

Fig. 8. X-ray photons from comet Hyakutake observed by the ROSAT Wide field camera on March 27.57, 1996. The contours are for 2, 4, 6 counts/pixel.

the instrumental background and by diffuse X-ray background radiation. The known X-ray sources can be subtracted from the observed photons. But a background radiation remains, which must be taken into account. We assume here for simplicity that the background is constant over the field of view. This assumption is approximately satisfied for most of the observations (see
Sect. 9 for an exception). Therefore, we make for the total surface intensity the more general ansatz

$J(\boldsymbol{x})=I_{\mathrm{back}}+\frac{H}{H_{0}} \cdot J_{\alpha}\left(\frac{R_{S, 0}}{R_{\mathrm{S}}} x\right)$

where $I_{\text {back }}$ is the background intensity, $H$ is the solar wind heavy ion energy flux density (defined in Eq. (7)), $R_{\mathrm{S}}$ is the shock distance. $H_{0}$ and $R_{\mathrm{S}, 0}$ are the solar wind heavy ion energy flux density and the shock distance of a reference model comet. Here we have used the scaling with shock distances instead of $L_{x}$. We could equally well use the scaling with production rates $G$.

The function $J$ still contains three unknown parameters, namely $I_{\text {back }}, H$, and $R_{\mathrm{S}}$. We can use the condition that the total energy emitted from the model comet in the field of view $V$ in the exposure time $T$ must be equal to the total observed energy $E_{\text {tot,obs }}$ in the same field. Therefore, $p(\boldsymbol{x})=$ $J(\boldsymbol{x}) T(\boldsymbol{x}) / E_{\text {tot,obs }}$ is a probability density in the region $V$.

We define the parameters

$\theta_{1}=\frac{I_{\text {back }}}{E_{\text {tot,obs }}}, \quad \theta_{2}=\frac{1}{E_{\text {tot, } m o d}}\left(1-\theta_{1} \int_{V} T(\boldsymbol{x}) \mathrm{d} x \mathrm{~d} y\right)$,

$\delta=\frac{R_{\mathrm{S}, 0}}{R_{\mathrm{S}}}$

where

$E_{\mathrm{tot}, \mathrm{mod}}=\int_{V} J_{\alpha}(\delta \cdot \boldsymbol{x}) T(\boldsymbol{x}) \mathrm{d} x \mathrm{~d} y$ 

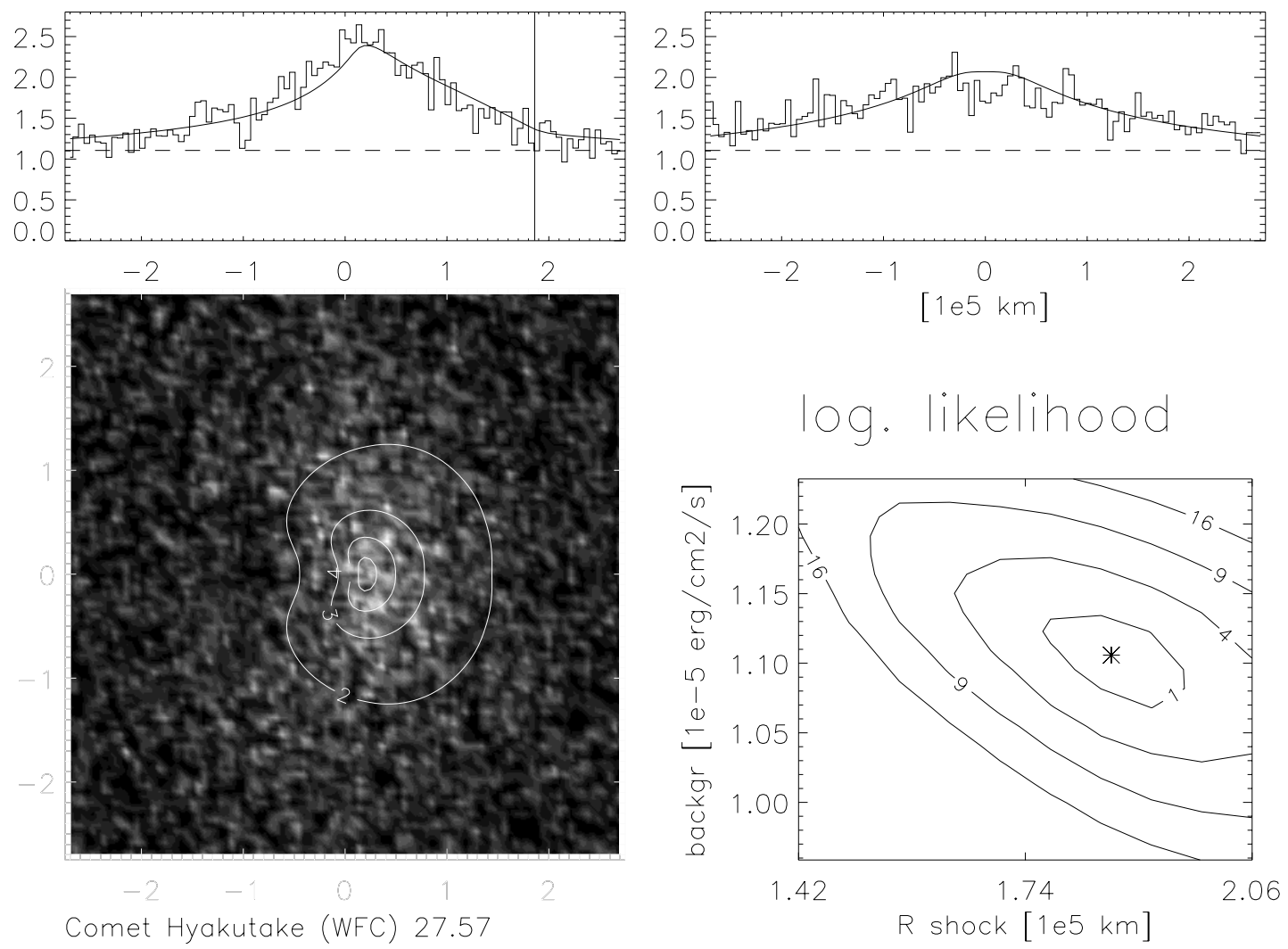

Fig. 9. Analysis of the photons of Fig. 8. Lower right: contours of the likelihood as a function of $R_{\mathrm{S}}$ and $I_{\text {back. }}$ The star marks the maximum. The contours are boundaries of confidence regions. The values are with probability $1-\mathrm{e}^{-k}$ inside the contour labeled $k$. Lower left: observed intensity (shades) and the intensity of the best adapted model (contours). Top panels: intensities (units: $10^{-5} \mathrm{erg} \mathrm{s}^{-1} \mathrm{~cm}^{-2}$ ) averaged over lines perpendicular to the Comet-Sun line (left) and parallel (right). The solid lines are from the model, the histograms from the data. The dashed line is the background. The vertical line in the upper left panel marks the shock position.

is the total energy of the (geometrically scaled) model emitted in the time $T$. Then the probability density can be written as

$p(\boldsymbol{x})=\theta_{1} T(\boldsymbol{x})+\theta_{2} J_{\alpha}(\delta \cdot \boldsymbol{x}) T(\boldsymbol{x})$

depending on two parameters $\theta_{1}$ and $\delta$.

When $n_{i}$ photons are observed at positions $\boldsymbol{x}_{i}$ during an exposure time $T\left(\boldsymbol{x}_{i}\right)$ one can form the (logarithmic) likelihood

$L\left(\theta_{1}, \delta\right):=\sum_{i} n_{i} \log p\left(\boldsymbol{x}_{i}\right)$

where the sum is over all pixels. One can get an estimate for $\theta_{1}$ and $\delta$ by maximizing the likelihood (26) as a function of these two parameters. In view of the relations (23) one can express the likelihood function also in the variables $I_{\text {back }}$ and $R_{\mathrm{S}}$. We use these variables in the following analyses since these are the physical quantities of interest.

The solar wind heavy ion energy flux density (as a fraction of the model flux) is determined in terms of the parameter $\theta_{2}$ as

$$
\frac{H}{H_{0}}=\theta_{2} E_{\text {tot,obs. }} \text {. }
$$

Near the maximum the function $L\left(\theta_{1}, \delta\right)$ is well described by a constant minus a positive definite quadratic function in the two variables $\theta_{1}$ and $\delta$. The maximum likelihood estimators for these parameters are for large samples asymptotically
Gaussian distributed with this quadratic function in the exponential. Therefore the contour lines of the likelihood function $L$ are boundaries of confidence regions of the estimates (see e.g. Brandt 1998).

We estimate two parameters, namely $R_{\mathrm{S}}$ and $I_{\mathrm{back}}$. Each estimate has its standard deviation $\sigma$. The estimates are correlated. Therefore, the ellipses in the likelihood contours (see e.g. the lower right panel of Fig. 9) are inclined with respect to the axes. The total variation of the estimate for $R_{\mathrm{S}}$ is not only determined by the value of its $\sigma$ but also to some extent by the error in the estimate for $I_{\text {back. }}$. In what follows we give only the individual $\sigma$ as error bars. Improved error estimates can be derived from the contours in the likelihood plots, which are at the same time confidence regions for the pair $R_{\mathrm{S}}$ and $I_{\mathrm{back}}$ of estimates.

\section{Analysis of the observations}

Here we discuss our model analysis of the X-ray observations of four comets.

\subsection{Comet Hyakutake, WFC observations}

We have analyzed the photons from comet Hyakutake in the ROSAT Wide Field Camera in a field $V$ of $100 \times 100$ pixels around the nucleus. This is the square in Fig. 8. This field 

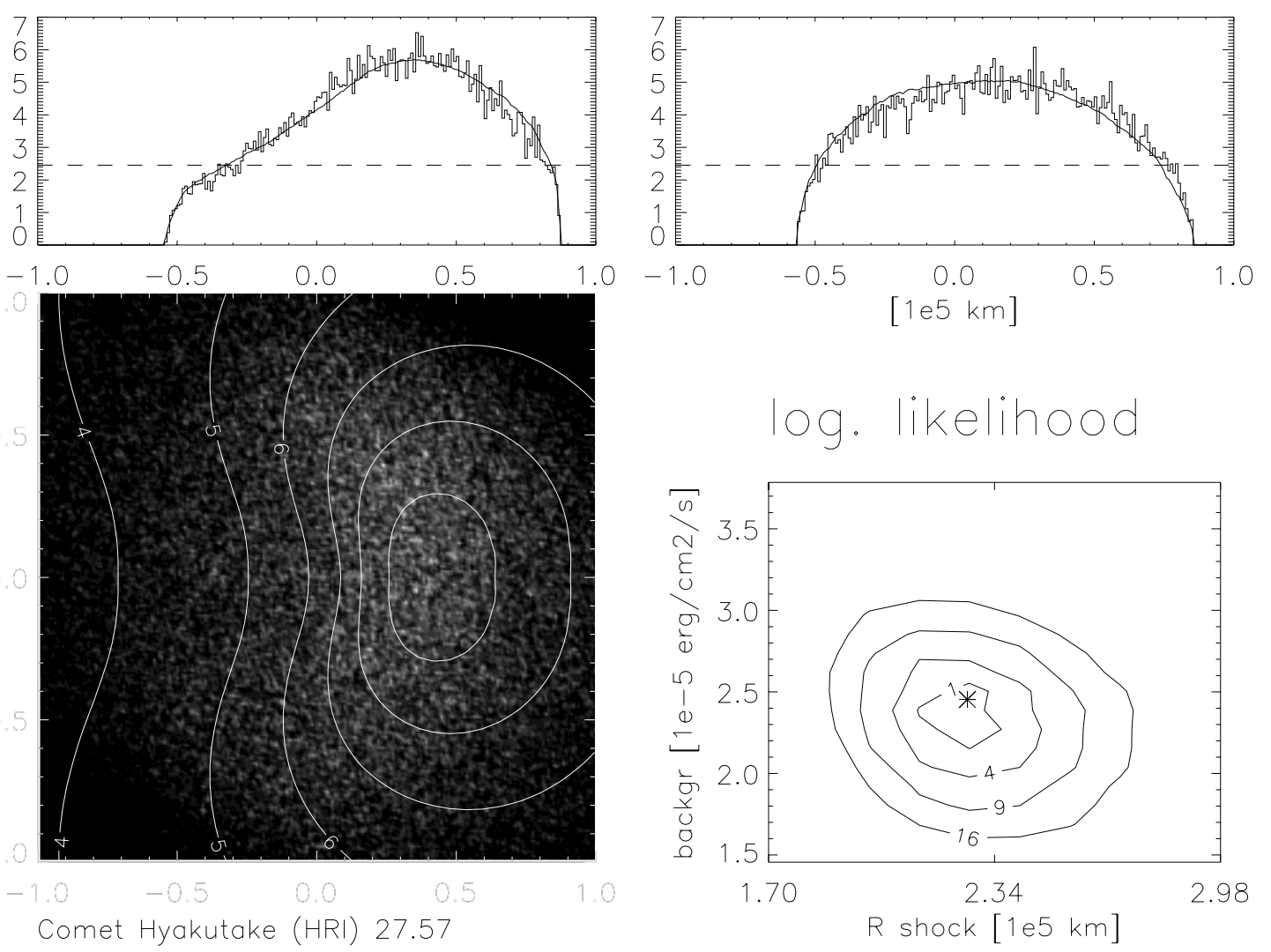

Fig. 10. The same as Fig. 9 for the analysis of the photons registered by HRI on May 27.571996 from comet Hyakutake.

covers $2.73 \times 10^{5} \mathrm{~km}$ on each side of the nucleus. In order to choose an appropriate model we note that the observations were done with an instrument which is sensitive only in the range from 90 to $206 \mathrm{eV}$ (Lisse et al. 1996). We use a reference model with a production $G_{0}=10^{29} \mathrm{~s}^{-1}$. Since Earth was in slow solar wind at that time and Hyakutake was close $(\Delta=0.1 \mathrm{AU})$ to Earth we assume slow solar wind at the comet. The solar wind heavy ion energy flux in the model is $H_{0}=1.55 \times 10^{-4} \mathrm{erg} \mathrm{cm}^{-2} \mathrm{~s}^{-1}$. We take into account only the emission of photons with energies less than $200 \mathrm{eV}$. This model has shock distance $1.42 \times 10^{5} \mathrm{~km}$. The phase angle is $89^{\circ}$.

Figure 9 shows the results of the analysis. The likelihood function (lower right panel of Fig. 9) has a clear maximum which gives with one sigma error the estimates for the shock distance $R_{\mathrm{S}}=1.86 \pm 0.11 \times 10^{5} \mathrm{~km}$ and for the background $I_{\text {back }}=1.11 \pm 0.01 \times 10^{-5} \mathrm{erg} \mathrm{s}^{-1} \mathrm{~cm}^{-2}$. The shock distance corresponds to a production rate of $G=1.31 \pm 0.08 \times 10^{29} \mathrm{~s}^{-1}$. The contours of the likelihood functions are scaled so that with $63 \%$ probability the values are inside the innermost contour, and $98 \%$ probability inside the second contour. For the WFC data we get a solar wind heavy ion energy flux $H=$ $0.88 H_{0}$. In order to infer from the $\leq 200 \mathrm{eV}$ photons the total solar wind flux we assumed that the soft photons comprise about $40 \%$ of the total intensity. The agreement of the observed photon distribution with the intensities from a model with the parameters determined by the maximum likelihood method is very good. This is most convincingly demonstrated by the intensities averaged over the $x$ and $y$ coordinates.

\subsection{Comet Hyakutake, HRI observations}

We have analyzed the $220 \times 220$ central pixels of the Hyakutake HRI data from comet Hyakutake which occupy a range of $10^{5} \mathrm{~km}$ on each side of the nucleus. The field of view is an oval (see the lower left panel of Fig. 10), so we have to use this oval as the region $V$ of the analysis. The field of view is rather small with the edge inside the bow shock. There are 37216 photons in this field. We analyze the photons with a reference model with a production $G_{0}=10^{29} \mathrm{~s}^{-1}$, and slow solar wind. Contrary to what has been done for the WFC data, we use in the model the full energy output in $\mathrm{X}$-rays. Figure 10 shows the main results. The likelihood function peaks at a shock distance $R_{\mathrm{S}}=2.27 \pm 0.09 \times 10^{5} \mathrm{~km}$ which corresponds to a production rate $G=1.60 \pm 0.06 \times 10^{29} \mathrm{~s}^{-1}$. The estimated intensity of the background is $I_{\mathrm{back}}=2.45 \pm$ $0.05 \times 10^{-5} \mathrm{erg} \mathrm{s}^{-1} \mathrm{~cm}^{-2}$. The solar wind heavy ion energy flux is $72 \pm 2 \%$ of that used in the model. Figure 10 also shows the comparison of the observed photon distribution with the best adapted model. The agreement is very good.

\subsection{Comet Levy}

Photons from Comet Levy on Sept. 61990 have been found in the ROSAT All-Sky Survey which was obtained with the PSPC instrument (Dennerl et al. 1997). These data provide an example of a comet observed under a phase angle $52^{\circ}$, significantly different from the typical $90^{\circ}$. We take for analysis 

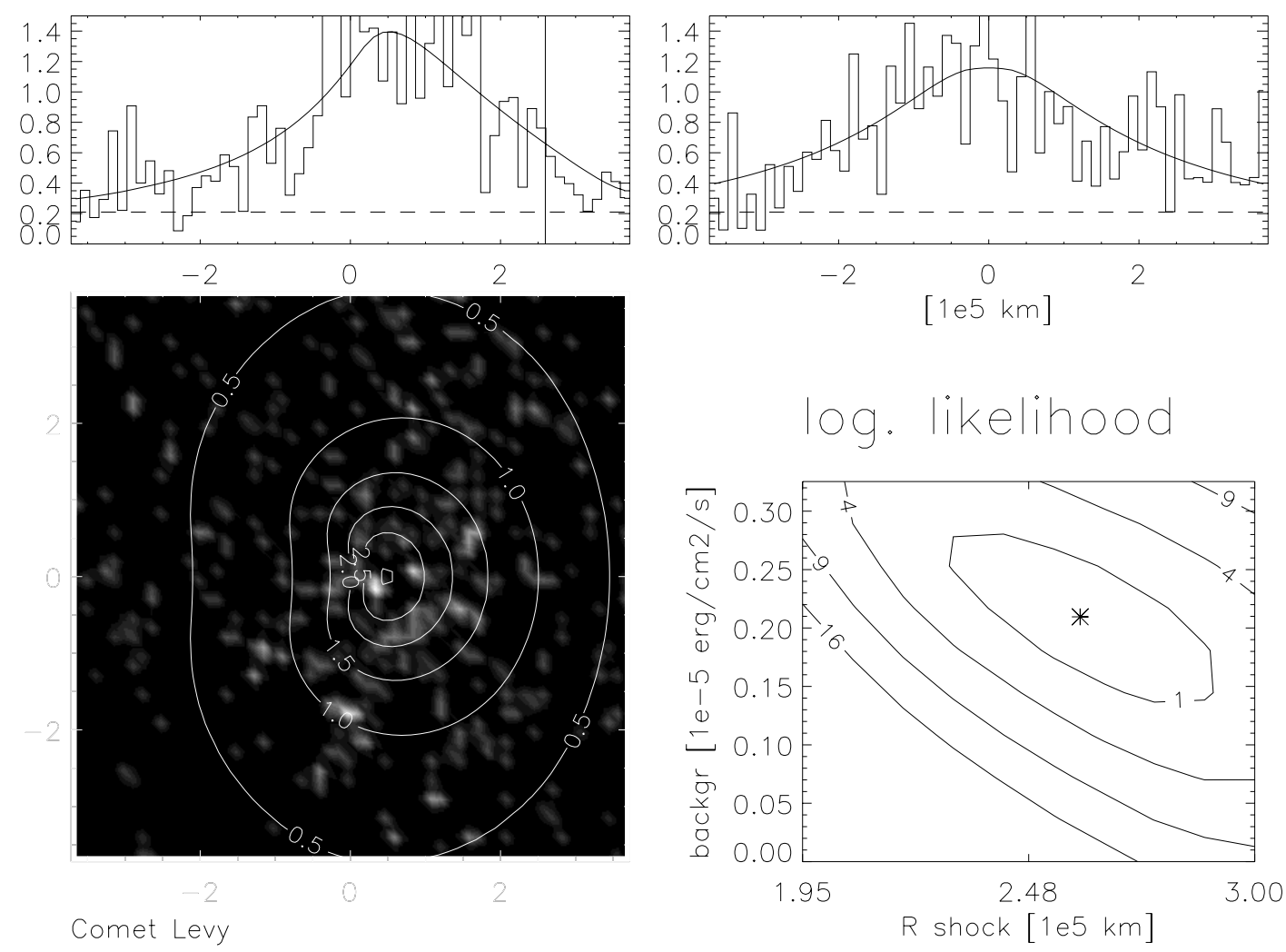

Fig. 11. The same as Fig. 9 for the analysis of the photons registered by ROSAT in the All-Sky Survey from comet Levy.

the photons from the central $60 \times 60$ pixels which cover a field of $3.7 \times 10^{5} \mathrm{~km}$ on each side of the nucleus. There are $391 \mathrm{X}$-ray photons in this field. We compare it with a model with $G_{0}=3 \times 10^{29} \mathrm{~s}^{-1}$ and slow solar wind, observed under the angle $\alpha=52^{\circ}$. Maximum likelihood analysis gives a shock distance $R_{\mathrm{S}}=2.60 \pm 0.17 \times 10^{5} \mathrm{~km}$ and a production rate $G=2.00 \pm 0.13 \times 10^{29} \mathrm{~s}^{-1}$. The background intensity is $I_{\text {back }}=2.10 \pm 0.65 \times 10^{-6} \mathrm{erg} \mathrm{s}^{-1} \mathrm{~cm}^{-2}$. Due to this low background $72 \%$ of the observed photons in the field are of cometary origin. The solar wind heavy ion energy flux is $H=0.38 \pm 0.09 H_{0}$ of the slow solar wind assumed in the reference model. Figure 11 shows the likelihood contours and the comparison of the observed photon distribution with the best adapted model.

Schleicher et al. (1991) have determined the OH production rate $Q(\mathrm{OH})$ for comet Levy at several dates by photometric methods. For Aug. 29.24 they obtain the value $Q(\mathrm{OH})=$ $2.3 \times 10^{29}$, and for Sept. 9.14 the value $Q(\mathrm{OH})=2.0 \times 10^{29}$ (Schleicher et al. 1991, Table II).

\subsection{Comet Encke}

Comet Encke is a low activity comet. The comet nevertheless left a clear signal of X-rays on a comparatively intense background (Lisse et al. 1999). The data were obtained with ROSAT HRI. We analyze the sum of all photons observed during the five days 4-8 July 1997. As reference we take a model with $G_{0}=10^{28} \mathrm{~s}^{-1}$ and slow solar wind, which corresponds to $H_{0}=1.55 \times 10^{-4} \mathrm{erg} \mathrm{cm}^{-2} \mathrm{~s}^{-1}$. The result of the analysis is shown in Fig. 12. The shock distance is only
$16700 \pm 460 \mathrm{~km}$. Nevertheless the maximum of the radiation comes from well inside the bow shock. The gas production is estimated to be $9.89 \pm 0.27 \times 10^{27} \mathrm{~s}^{-1}$.

The analysis of Lisse et al. (1999) started from the value $G=2 \times 10^{27}$ and derived a shock distance $R_{\mathrm{S}}=750 \mathrm{~km}$. The assumed value of $G$ is certainly too low by a factor of about four (see Mäkinen et al. 2001), and the shock distance is too low by an even larger factor.

The estimated background $I_{\text {back }}=2.03 \pm 0.03 \times$ $10^{-5} \mathrm{erg} \mathrm{s}^{-1} \mathrm{~cm}^{-2}$ comes close to the value of $1.7 \times$ $10^{-5} \mathrm{erg} \mathrm{s}^{-1} \mathrm{~cm}^{-2}$ given by the observers (see also Fig. 18). The solar wind is $0.338 \pm 0.006$ of the model value.

Figure 13 shows the average intensity $\bar{J}(R)$ of the observed $\mathrm{X}$-ray emission from comet Encke along rings of radius $R$ centered at the nucleus. Even far outside the bow shock the intensity is higher than the background. Outside the shock the simple model of Sect. 2 can be applied to predict a cometary emission of order $1 / R$. We fitted a function

$\bar{J}(R) \approx I_{\mathrm{back}}+\frac{A}{R}$

to the data. By a least squares fit we determined the background $I_{\text {back }}=1.94 \times 10^{-5} \mathrm{erg} \mathrm{s}^{-1} \mathrm{~cm}^{-2}$ and the constant $A=10900$. With the values from the estimate above we calculate the right hand side of Eq. (11) for comet Encke, $H G Q / 4 w=6500$, which is of the same order as the constant $A$ determined before. This demonstrates that outside the bow shock photons are also generated by charge exchange of solar wind heavy ions with cometary gas molecules. 

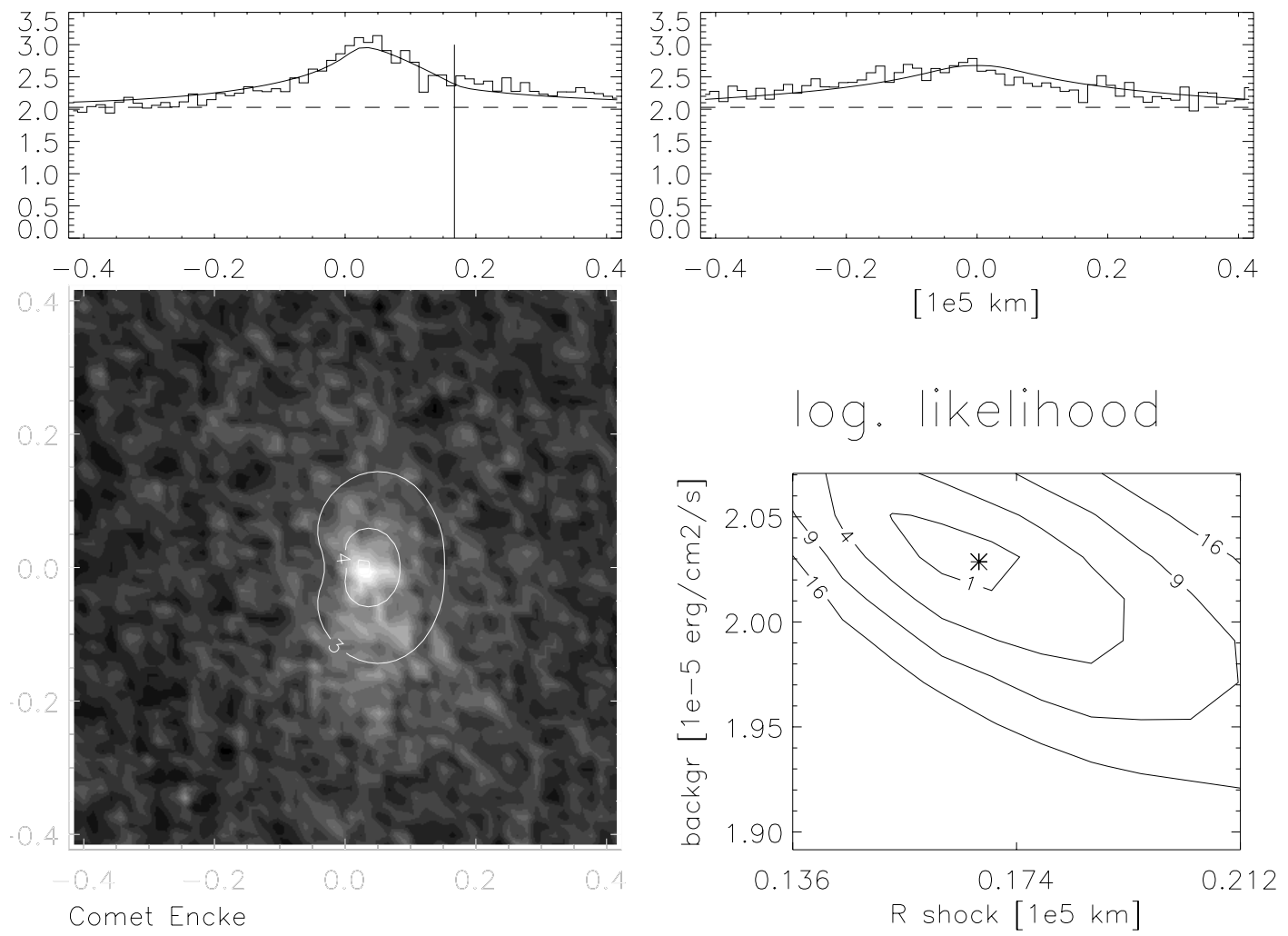

Fig. 12. The same as Fig. 9 for the analysis of the photons registered by ROSAT from comet Encke.

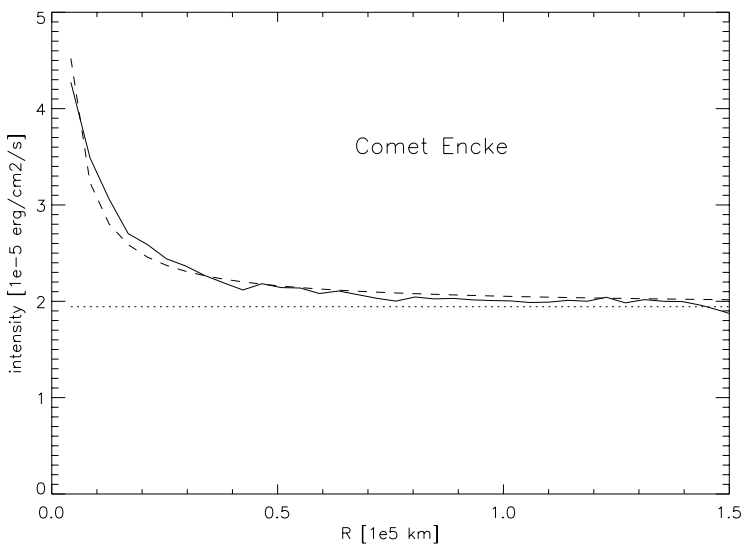

Fig. 13. The observed intensity $\bar{J}(R)$ averaged along rings of radius $R$ from comet Encke (solid line), the approximation (28) (dashed) and the background $I_{\text {back }}$ (dotted).

\subsection{Comet C/2000 WM1 (LINEAR)}

As an example of a comet observed with the new generation of X-ray satellites we consider Comet C/2000 WM1 (LINEAR). This comet was observed on 2001 December 13/14 with XMM-Newton and more than a million photons were recorded (Dennerl et al. 2003). The phase angle was $74^{\circ}$. We have analyzed the data with a reference model of $G_{0}=10^{29} \mathrm{~s}^{-1} \mathrm{ob}-$ served at $74^{\circ}$. The comet was at low heliographic latitude $\approx-9^{\circ}$. But there are reasons to believe that the comet was in fast solar wind (Brandt 2002). The result is shown in Fig. 14. The estimated shock distance is $77000 \pm 200 \mathrm{~km}$ which corresponds to a total gas production of $G=7.14 \pm 0.02 \times 10^{28} \mathrm{~s}^{-1}$. This value agrees well with the water production rate of $5.2 \times 10^{28} \mathrm{~s}^{-1}$ determined by Schleicher et al. (2002) on December 13. The estimated solar wind flux is $1.7 H_{0}$. The high photon number yields infinitesimal statistical errors. The systematic errors caused by uncertainties in the model assumptions (e.g. crosssections, state of the captured electrons, de-excitation mechanism etc.) are much larger. Dennerl et al. (2003) find that the surface brightness of Comet C/2000 WM1 (LINEAR) decreases with $1 / R$ with increasing distance from the nucleus. This agrees very well with our theoretical prediction (10).

\section{Analysis of the variability}

\subsection{Comet Hyakutake}

Cometary X-ray emission is also highly variable. Excursions of factors of up to three have been reported by a number of authors (e.g. Lisse et al. 1996, 1999; Neugebauer et al. 2000). Here we analyze the data from comet Hyakutake for the nine dates listed by Lisse et al. (1996). Since our analysis gives independent measures of the gas production and the solar wind heavy ion flux, we can infer from our results where the variability comes from.

In Fig. 15 we have inserted the gas production rates obtained by our method for the data from the WFC and the HRI instrument. Most estimates are obtained with the slow solar wind reference model. For a few dates we have also determined values with a fast solar wind model. For comparison we have added the results obtained from observations at longer wavelength, namely the observation of $\mathrm{L} \alpha$ by SOHO/SWAN by Bertaux et al. (1998) and the radio observation of the 

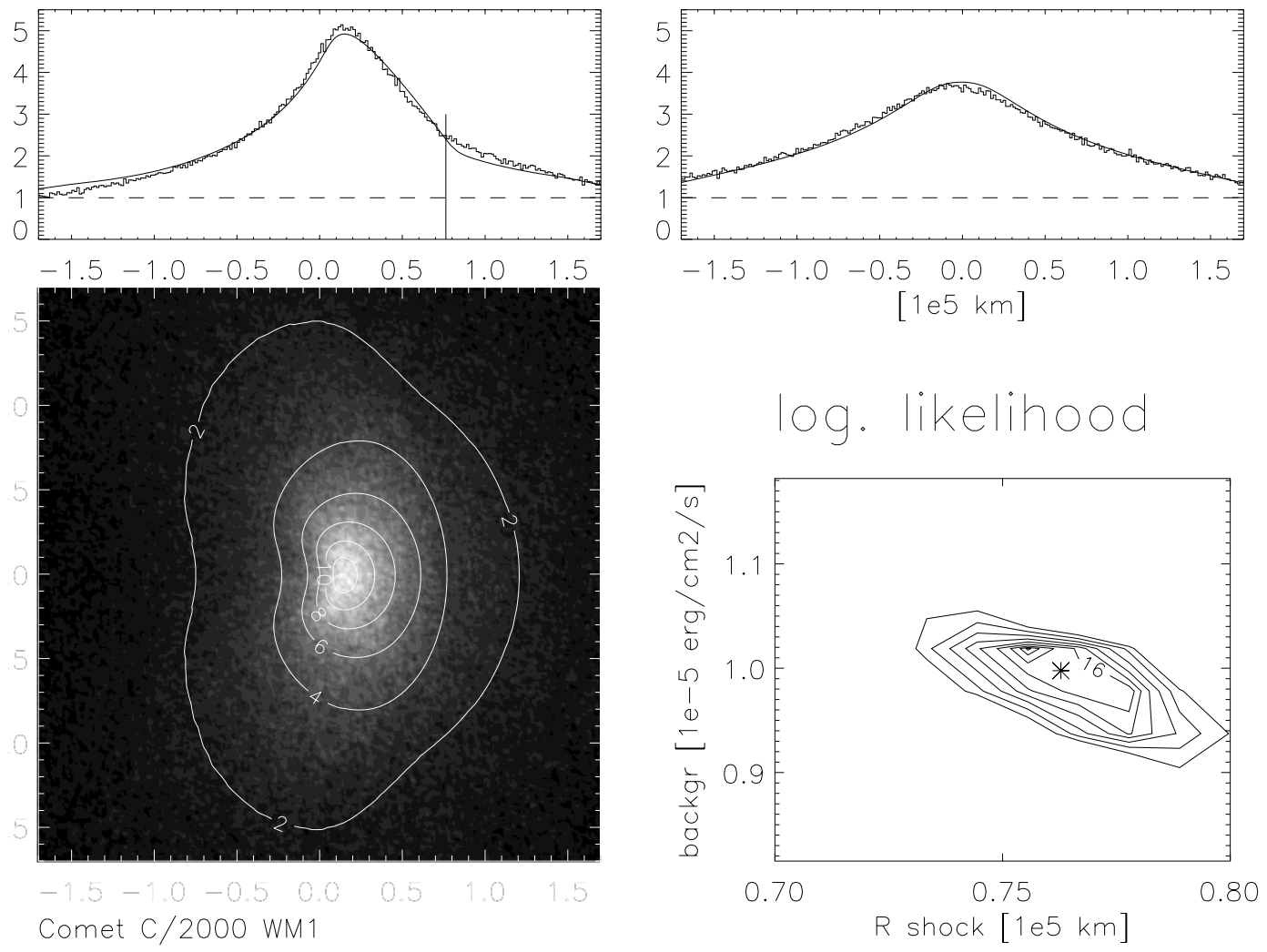

Comet C/2000 WM1

Fig. 14. The same as Fig. 9 for the analysis of the photons registered by XMM-Newton from comet C/2000 WM1 (LINEAR).

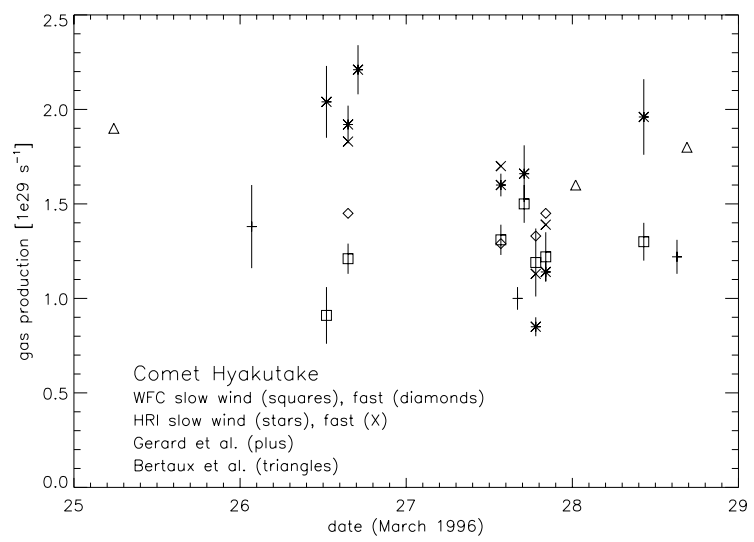

Fig. 15. Gas production rates determined from the X-ray observations of comet Hyakutake, and other published values for the same time.

18-cm line of $\mathrm{OH}$ by Gérard et al. (1998). The agreement is rather good. To give an impression of the accuracy of the statistical method we have inserted a few one sigma error bars. Our results are consistent with the range of other published results.

In Fig. 16 we have plotted the solar wind heavy ion energy flux determined from the nine X-ray observations of comet Hyakutake in March 1996. The figure shows the fraction $H / H_{0}$ of the flux in the best adapted model and the solar wind assumed in the model. For two dates the signal is too low for analysis.

Near-Earth solar wind data compiled from several spacecraft are available via the World Wide Web from the National Space Science Data Center OMNIWeb

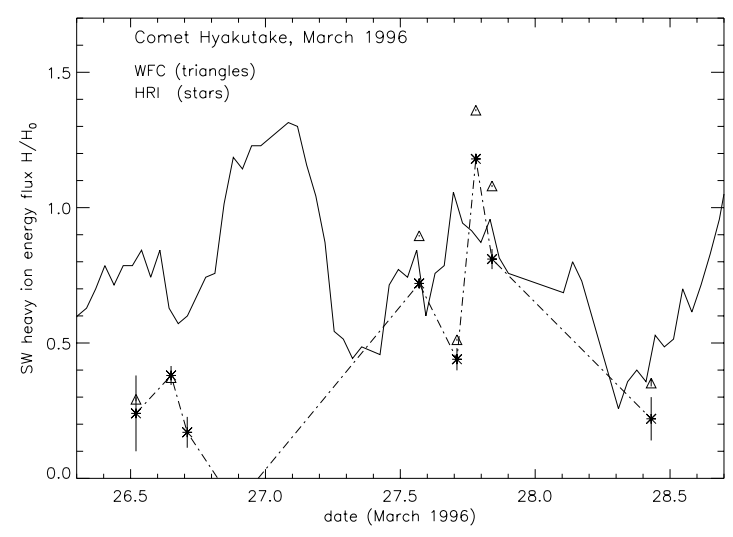

Fig. 16. The solar wind heavy ion energy flux as a function of time determined from the X-ray observations of comet Hyakutake. The solid line is the observed solar wind proton density divided by 7 from the Omniweb and shifted to the comet's position following Neugebauer et al. (2000).

(http://nssdc.gsfc.nasa.gov/omniweb). We have added the solar wind proton densities from the Omniweb shifted to the comet's position as described by Neugebauer et al. (2000). The proton densities in Fig. 16 are reduced by a factor of 7 to the assumed proton density $n_{\mathrm{p}}$ in the reference model. In the reference model we have assumed a solar wind oxygen/hydrogen ratio of 0.0005 . From the ratio $H / n_{\mathrm{p}}$ of the estimated solar wind heavy energy flux and the normalized solar wind proton density in Fig. 16 we derive an estimate $0.0005 \times H / n_{\mathrm{p}}$ for the actual $[\mathrm{O} / \mathrm{H}]$ ratio. In the first part of the observations this ratio is about 0.0002 , in the second part it is 


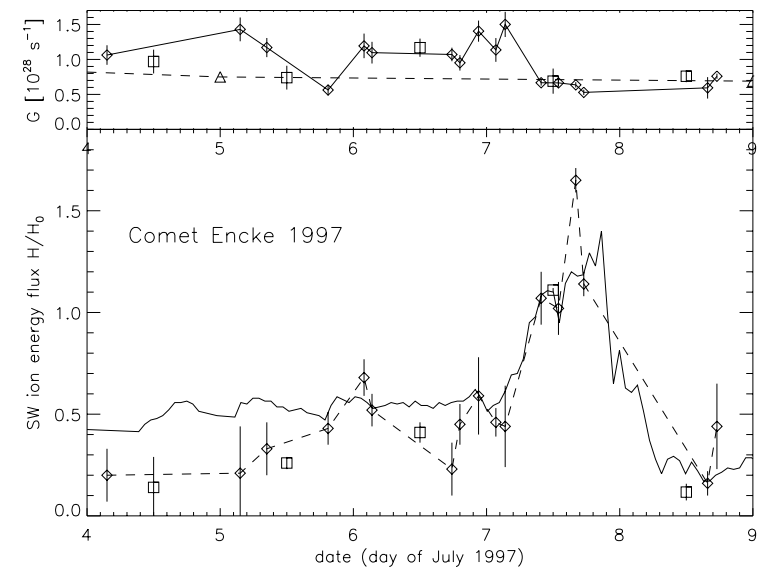

Fig. 17. Lower panel: the solar wind heavy ion energy flux with statistical errors determined from the X-ray observations of comet Encke as a function of time (diamonds and dashed line). The solid line is the solar wind proton density divided by 14 from the Omniweb shifted to the comet's position. Upper panel: the estimated gas production (diamonds and solid line) with error bars, and the results of Mäkinen et al. (2001) (triangle and dashed line). The squares mark estimates from all photons of the day.

higher than this value by a factor of $2-3$. This indicates that the observed X-ray outburst is mainly caused by an increase of the $[\mathrm{O} / \mathrm{H}]$ ratio, as concluded by Neugebauer et al. (2000), rather than an increase in the overall solar wind flux density.

\subsection{Comet Encke}

We have also analyzed the 1997 Encke ROSAT data for several dates where the comet's signal was sufficiently large.

The lower panel of Fig. 17 shows our estimates for the solar wind heavy ion energy flux (relative to the model value) and the proton densities from the Omniweb shifted to the position of the comet. The outburst in X-rays on July 7 is clearly related to the increase of the solar wind proton density (using the solar wind proton flux as a proxy) on the same day (see also Fig. 3b in Lisse et al. 1999). The estimated $[\mathrm{O} / \mathrm{H}]$ ratio is around .0001 before July 6 and .00025 after.

The upper panel of Fig. 17 shows the estimated gas production rate. It is around $G=1.1 \times 10^{28}$ from July 4 to July 7 . It drops to $0.7 \times 10^{28}$ during July 7 and stays at this value until the end of the series on July 8.73. It is remarkable that the $\mathrm{X}$-ray outburst occurred while the gas production was reduced by a factor of more than 1.5. Mäkinen et al. (2001) derive from SWAN observations water production rates of $1.03 \pm 0.07 \times 10^{28}$ at July 1 and $0.75 \pm 0.07 \times 10^{28}$ at July 5 . In their results there seems to be a discontinuous transition from high to low values during the period between July 1 and July 5. This is quite similar to our results.

\section{Background}

In Fig. 18 we compare the intensity of the X-ray background determined by the observers with our estimates obtained by the method of Sect. 6. Data are given for the nine observations of comet Hyakutake and for fifteen Encke data. The agreement

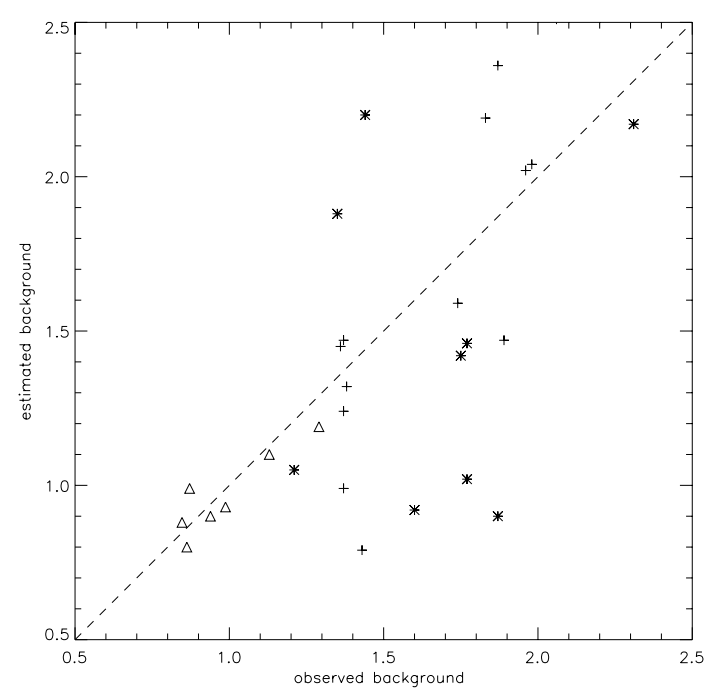

Fig. 18. Comparison of the intensity of the background radiation determined by the observers and estimated by the analysis of this paper. Hyakutake WFC (triangles), Hyakutake HRI (stars) and Encke (crosses). The unit is $10^{-5} \mathrm{erg} \mathrm{s}^{-1} \mathrm{~cm}^{-2}$.

is quite good with the exception of the HRI data of comet Hyakutake. Since comet Hyakutake fills the field of view of the HRI instrument completely, it is very difficult to determine the background by the usual method from the images. Therefore, the observers' values are likely to be rather inaccurate for the HRI data. On the other hand, for the HRI data the background is not constant over the field of view, so that one of the assumptions for our statistical analysis (see Sect. 6) is not satisfied. Therefore, our estimates for HRI are also not very reliable.

It is important to note here that the photon and background data are in units of counts per pixel per second. Assuming a source spectrum, one can transform this information into units of $\mathrm{erg} / \mathrm{cm}^{2} / \mathrm{s}$. Strictly speaking, this can be applied only to the counts of cometary X-ray photons. It cannot be applied to the instrumental background, which includes counts due to particle-induced events in the detector. The unit erg s $\mathrm{s}^{-1} \mathrm{~cm}^{-2}$ is therefore not quite appropriate. Nevertheless, we have used it, since it gives the best means to compare the contribution of the cometary X-rays with the background.

\section{Total luminosity}

Since the surface intensity of cometary X-ray emission is inversely proportional to the distance $R$ from the nucleus (see Eq. (10)) the total X-ray luminosity of a comet is infinite (at least in the idealized situation of our models). To get a reasonable measure for the total luminosity one has to sum the intensity $J_{\alpha}$ from a finite region $V$

$J_{\mathrm{tot}}=\int_{V} J_{\alpha}(\boldsymbol{x}) \mathrm{d} x \mathrm{~d} y$

This region can be the field of view of the detector. This gives a region $V$ which is not related to the comet. We discuss here only total luminosities determined from regions $V$ which are connected with the comet. Such a region is e.g. the region where the surface brightness is $\geq$ a fixed fraction of the peak 


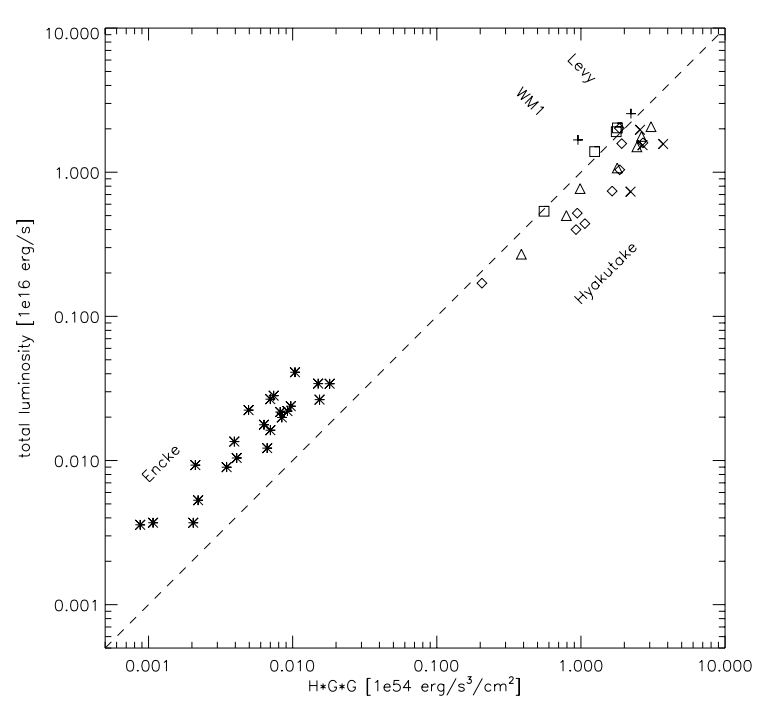

Fig. 19. The relation between the total $X$-ray luminosity and the quantity $H \cdot G^{2}$ for the comets Hyakutake: WFC (triangles), WFC fast wind (squares), HRI (diamonds) and HRI fast wind $(\times)$, Levy $(+)$, C/2000 WM1 (+) and Encke (*). The dashed line is relation (30).

brightness (e.g. Dennerl et al. 1997). It is apparent from Fig. 4 that with such a criterion the larger part of the total luminosity comes from inside the bow shock. In our analysis we use the computational grid of the model whose size is related to the shock distance.

In our analysis we can separate the contribution of the comet from the background and so determine the total luminosity of the comet. In Sect. 3 we derived a scaling law with length $L_{x}$. This is approximately also a scaling with the gas production $G$. If such a scaling holds, then the region $V$ of integration $V=G / G_{0} \cdot V_{0}$ is similar to a fixed region $V_{0}$, and the intensity $J_{\alpha}(\boldsymbol{x})=H / H_{0} \cdot J_{\alpha, 0}\left(G / G_{0} \cdot \boldsymbol{x}\right)$ is proportional to a scaled universal function $J_{\alpha, 0}$. The total luminosity $J_{\text {tot }}$ of the comet

$J_{\text {tot }}=\int_{V} J_{\alpha}(\boldsymbol{x}) \mathrm{d} x \mathrm{~d} y=H G^{2} \int_{V_{0}} J_{\alpha, 0}(\boldsymbol{x}) \mathrm{d} x \mathrm{~d} y=C \cdot H \cdot G^{2}$

is therefore proportional to $H \cdot G^{2}$. In Fig. 19 we have plotted the total luminosity of the comet, determined from the best fitted model, over the corresponding values of $H \cdot G^{2}$. This figure confirms the approximate relation (30) with $C=10^{-38} \mathrm{~s}^{2} \mathrm{~cm}^{2}$ over four orders of magnitude. The constant $C$ of proportionality in Eq. (30) depends on the spatial region $V$ over which the intensity is integrated. Note that the total luminosities of comet Hyakutake are underestimated, since HRI does not see the whole comet, and WFC measures only the low energy part $(<206 \mathrm{eV})$ of the energy spectrum.

\section{Conclusions}

Cometary X-rays are a good sensor for the solar wind heavy ion flux and provide a good means to measure the gas production rate of the comet. The spatial morphology of the X-ray emission depends mainly on the gas production rate, while the emission intensity is proportional to the heavy ion energy flux $H$ in the solar wind. In this paper, we have demonstrated how one can exploit these dependencies to determine by a statistical method from the observed X-ray photons the gas production rate $G$ of the comet, the heavy ion energy flux $H$ in the solar wind, and the diffuse X-ray background separately.

We have derived several length scales which govern the morphology of the X-ray emission of a comet. By comparing these length scales with hydrodynamic scales one can conclude that the maximum emission with its well known crescent shape occurs well inside the bow shock. There is, however, also cometary X-ray emission from far outside the bow shock with a surface intensity inversely proportional to the distance from the nucleus. This part of the radiation can be identified in wide field $\mathrm{X}$-ray images of comets. In our analysis of the Hyakutake and the Encke data we found as most likely causes of the observed variability changes in the $[\mathrm{O} / \mathrm{H}]$ ratio for comet Hyakutake and a solar wind density enhancement for comet Encke.

When the comet is close to Earth, reliable solar wind proton fluxes at the comet can be obtained from monitoring spacecraft. From our calculated flux of heavy ions, $H$, we then calculated the $[\mathrm{O} / \mathrm{H}]$ ratio in the solar wind at the time of the X-ray observation.

Comparison of the background derived from our method of morphological analysis and the results of imaging data analysis by various observers showed good agreement. Finally, we found an empirical relation showing that the total X-ray luminosity is proportional to the solar wind heavy ion energy flux $H$ and to the square of the gas production rate $G$ of the comet.

The ideas about the morphology of the cometary X-ray emission developed in this paper are based on model calculations which use a continuum description of the cometary plasma flow as well as of the neutral gas coma. These ideas have been successfully tested for comets with gas production rates $>7 \times 10^{27} \mathrm{~s}^{-1}$. We must be aware, however, that for comets with low gas production the scales are so small that plasmaphysical microprocesses had not time to relax, and a macroscopic description becomes inadequate. This problem has been discussed in the context of the encounter of the spacecraft Giotto with Comet P/Grigg-Skjellerup which had at that time a gas production of about $4 \times 10^{27} \mathrm{~s}^{-1}$ (see e.g. Schmidt et al. 1993).

\section{References}

Bertaux, J. P., Costa, J., Quémerais, E., et al. 1998, Planet. Space Sci., 46, 555

Brandt, S. 1997, Data analysis (Springer)

Brandt, J. C., et al. 2002, Asteroids, Comets, Meteors, ESA-SP-500

Cravens, T. E. 1997, Geophys. Res. Lett., 24, 105

Dennerl, K., Englhauser, J., \& Trümper, J. 1997, Science, 277, 1625

Dennerl, K., Aschenbach, B., Burwitz, V., et al. 2003, in X-Ray and Gamma-Ray telescopes and instruments in astronomy, ed. J. E. Trümper, \& H. D. Tananbaum, Proc. SPIE, 4851

Gérard, E., Crovisier, J., Colom, P., et al. 1998, Planet. Space Sci., 46, 569

Häberli, R. M., Gombosi, T. I., De Zeeuw, D. L., Combi, M. R., \& Powell, K. G. 1997, Science, 276, 939

Kharchenko, V., \& Dalgarno, A. 2000, J. Geophys. Res., 105, 18351

Kharchenko, V., \& Dalgarno, A. 2001, ApJ, 554, L99

Krasnopolsky, V. A., Mumma, M. J., \& Abbott, M. J. 2000, Icarus, 146,152 
Krasnopolsky, V. A., Christian, D. J., Kharchenko, V., et al. 2002, Icarus, 160,437

Lisse, C. M., Dennerl, K., Englhauser, J., et al. 1996, Science, 274, 205

Lisse, C. M., Christian, D., Dennerl, K., et al. 1999, Icarus, 141, 316

Lisse, C. M., Christian, D. J., Dennerl, K., et al. 2001, Science, 292, 1343

Lisse, C. M., Cravens, T. E., \& Dennerl, K. 2004, X-ray and extreme ultraviolet emission from Comets, Comets II, in press

Mäkinen, J. T. T., Silén, J., Schmidt, W., et al. 2001, Icarus, 152, 268

Mumma, M. J., Krasnopolsky, V. A., \& Abbott, M. J. 1997, ApJ, 491, L125

Neugebauer, M., Cravens, T. E., Lisse, C. M., et al. 2000, J. Geophys. Res., 105, 20949
Owens, A., Parmar, A. N., Oosterbroek, T., et al. 1998, ApJ, 493, L47 Schleicher, D. G., Millis, R. L., Osip, D. J., \& Birch, P. V. 1991, Icarus, 94,511

Schleicher, D. G., Woodney, L. M., \& Birch, P. V. 2003, Earth, Moon and Planets, 90, 401

Schmidt, H. U., \& Wegmann, R. 1982, in Comets, ed. L. L. Wilkening (Tucson: Univ. Arizona Press), 538

Schmidt, H. U., Wegmann, R., \& Neubauer, F. M. 1993, J. Geophys. Res., 98, 21009

Schwadron, N. A., \& Cravens, T. E. 2000, ApJ, 544, 558

Wegmann, R., Schmidt, H. U., Lisse, C. M., Dennerl, K., \& Englhauser, J. 1998, Planet. Space Sci., 46, 603

Wegmann, R., Jockers, K., \& Bonev, T. 1999, Planet. Space Sci., 47, 745 